\title{
Analyst
}



Cite this: Analyst, 2014, 139, 6135

Received 20th August 2014

Accepted 9th October 2014

DOI: $10.1039 / c 4 a n 01523 a$

www.rsc.org/analyst

\section{Multimodal optical characterisation of collagen photodegradation by femtosecond infrared laser ablation $\uparrow$}

\author{
A. Manickavasagam, ${ }^{a}$ L. M. Hirvonen, ${ }^{a}$ L. N. Melita, ${ }^{C}$ E. Z. Chong, ${ }^{\text {a }}$ R. J. Cook, ${ }^{\text {ab }}$ \\ L. Bozec ${ }^{c}$ and F. Festy ${ }^{\star a}$
}

Collagen is a structural component of the human body, as a connective tissue it can become altered as a result of pathophysiological conditions. Although the collagen degradation mechanism is not fully understood, it plays an important role in ageing, disease progression and applications in therapeutic laser treatments. To fully understand the mechanism of collagen alteration, in our study photo-disruptive effects were induced in collagen I matrix by point-irradiation with a femtosecond Ti-sapphire laser under controlled laser ablation settings. This was followed by multi-modal imaging of the irradiated and surrounding areas to analyse the degradation mechanism. Our multi-modal methodology was based on second harmonic generation (SHG), scanning electron microscope (SEM), autofluorescence (AF) average intensities and the average fluorescence lifetime. This allowed us to quantitatively characterise the degraded area into four distinct zones: (1) depolymerised zone in the laser focal spot as indicated by the loss of SHG signal, (2) enhanced crosslinking zone in the inner boundary of the laser induced cavity as represented by the high fluorescence ring, (3) reduced crosslinking zone formed the outer boundary of the cavity as marked by the increased SHG signal and (4) native collagen. These identified distinct zones were in good agreement with the expected photochemical changes shown using Raman spectroscopy. In addition, imaging using polarisation-resolved SHG (p-SHG) revealed both a high degree of fibre reorientation and a SHG change in tensor ratios around the irradiation spot. Our multi-modal optical imaging approach can provide a new methodology for defining distinct zones that can be used in a clinical setting to determine suitable thresholds for applying safe laser treatments without affecting the surrounding tissues. Furthermore this technique can be extended to address challenges observed in collagen based tissue engineering and used as a minimally invasive diagnostic tool to characterise diseased and non-diseased collagen rich tissues.

\section{Introduction}

Collagen is a major fibrous protein present in all mammals, constituting $25 \%$ of the body's total protein volume. ${ }^{1}$ It is composed of three polypeptide $\alpha$ chains coiled around a central

${ }^{a}$ Biomaterial, Biomimetics \& Biophotonics Division, King's College London Dental Institute, London, UK. E-mail: frederic.festy@kcl.ac.uk; Tel: +44 (o)20 71880143 ${ }^{b}$ Oral Medicine, King's College London Dental Institute, London, UK

${ }^{c}$ Biomaterials \& Tissue Engineering Division, Eastman Dental Institute, University College London, London, UK

$\dagger$ Electronic supplementary information (ESI) available: Raman cluster analysis showing groups of dissimilar clusters identified. The cluster 1 represents depolymerised collagen (zone 1), cluster 2 attributes to the enhanced cross-linking zone (zone 2), cluster 3 corresponds to the reduced cross-linking zone (zone 3 ) and cluster 4 showing healthy collagen (zone 4), laser induced cavity evolution video obtained with time lapse imaging (field size $36 \mu \mathrm{m}$ ) showing the origin of the cavity, growth in cavity size and thermal stability. SHG (left), AF (centre) and combined result of SHG and AF imaging (right). See DOI: $10.1039 / \mathrm{c} 4 \mathrm{an} 01523 \mathrm{a}$ axis to form a triple helical structure, ${ }^{2}$ which is $\sim 300 \mathrm{~nm}$ in length and $\sim 1.5 \mathrm{~nm}$ in diameter, stabilised by intermolecular hydrogen and bound water molecules. ${ }^{3}$ Under physiological conditions, these molecules have the property to self-assemble to create a higher hierarchical structure known as the collagen fibril. Each fibril contains thousands of collagen molecules which in turn, is stabilised by the presence of intermolecular crosslinks. Interestingly, collagen fibrils display a banding periodicity of $\mathrm{D}=67 \mathrm{~nm}$ along their long axis arising from an internal organisation of the collagen molecules. ${ }^{4,5}$ To date, there are about 28 genetically distinct collagen types identified, based on the differences in their polypeptide sequence. ${ }^{6}$ As a connective tissue, fibrillar collagen provides structural support and mechanical stability to the animal body. ${ }^{7}$ This study focuses on type I collagen which is relatively abundant in skin, tendon, bone and cornea.

In certain pathophysiological conditions such as diabetes and cancers, thermal challenge and laser irradiation can be used as part of the treatment plan and they can cause collagen 
to undergo structural and compositional changes. ${ }^{3,8,9}$ Collagen degradation mechanisms are therefore subject to extensive studies due to the potential impacts of tissue function loss as a result such treatment plan. ${ }^{10}$ Furthermore, the rapid growth of laser therapeutic and diagnostic applications, combined with the extensive use of collagens in medicine, mandates an indepth understanding of laser-collagen interactions. ${ }^{11,12}$ In particular, it is important to understand the physical and chemical alterations of the collagen matrix during laser induced photodegradation. ${ }^{13}$

Collagen denaturation is a complex process which greatly depends on the collagen type and its degree of crosslinking. It can be defined in two ways: (1) unwinding of the triple helical structure of the fibril by the failure of the intramolecular crosslinks (thermal denaturation) or (2) sectioning of the $\alpha$ chains leading to the collapse of the triple helical structure (enzymatic degradation). Although denaturation may start occurring at the molecular level, its impact can be observed throughout the collagen's hierarchical structure. ${ }^{14}$

Previous studies have reported several techniques used in studying collagen structure such as differential scanning calorimetry (DSC), ${ }^{15} \mathrm{X}$-ray diffraction (XRD), ${ }^{15,16}$ scanning electron microscopy (SEM), ${ }^{17}$ mechanical examination, histological and biochemical assays. ${ }^{16}$ Unfortunately, these techniques cannot be readily used on patients and thus, optical imaging is the preferable choice as the diagnostic tool. Optical coherence tomography (OCT) has been effectively applied to imaging cross-sections of collagen rich tissues such as eyes ${ }^{18}$ and skin. ${ }^{19,20}$ Nonlinear optical imaging techniques based on twophoton excited fluorescence ${ }^{21}$ and second harmonic generation $(\mathrm{SHG})^{22}$ are the most favourable high resolution imaging techniques for studying collagen. They provide direct information on the collagen structures without the need for external staining agents, leading to a reduced phototoxicity. ${ }^{23}$ Furthermore, polarisation-resolved SHG (p-SHG) microscopy has been used as a quantitative tool for measuring collagen fiber type and orientation in tissues. ${ }^{22}$ To date, majority of the studies on collagen denaturation have only involved single imaging modality and a precise understanding of the physical and chemical alterations occurring during collagen degradation under controlled conditions are yet to be established. ${ }^{24}$ Unfortunately, few reports that have utilised multimodal imaging approach for collagen-rich tissue characterization were generally qualitative in nature. ${ }^{3,8,14,25-27}$ The primary aim of this study is to qualitatively and quantitatively characterise laser induced type I collagen denaturation using p-SHG, fluorescence lifetime imaging (FLIM), SEM and Raman spectroscopy. The degree of photodegradation from varying the irradiation parameters such as laser output power, excitation wavelength and irradiation time were measured starting from the laser irradiation spot.

\section{Materials and methods}

\subsection{Collagen scaffold}

Acellular collagen gels were made by mixing $4 \mathrm{~mL}$ of sterile acid soluble collagen solution $\left(2.05 \mathrm{mg} \mathrm{mL}^{-1}\right.$ protein in $0.6 \%$ acetic acid: First Link Ltd, West Midlands, UK) with $0.5 \mathrm{~mL}$ of
$10 \times$ concentration Minimum Essential Medium (MEM) (Gibco Chemicals, Invitrogen, UK). $5 \mathrm{M}$ and $1 \mathrm{M}$ sodium hydroxide $(\mathrm{NaOH})$ was added dropwise to the solution to reach $\mathrm{pH} \sim 7$. After neutralisation, the solution was cast into rectangular moulds $(22 \times 33 \times 10 \mathrm{~mm})$ and placed in an incubator at $37{ }^{\circ} \mathrm{C}$ for $30 \mathrm{~min}$ to allow gel formation. Following setting and incubation, hyperhydrated gels were plastically compressed (PC) to remove rapidly fluids, applying the PC protocol described in the literature by Brown et al. ${ }^{28}$ Gels were set between two nylon meshes and placed over a metal mesh and a double layer of filter paper. A $90 \mathrm{~g}$ metal load was placed on top of the gels and left to compress for $5 \mathrm{~min}$. The loss of fluids reduced the gel thickness from initially $\sim 50-80 \mu \mathrm{m}$ to approximately $\sim 4 \mu \mathrm{m}$. Collagen sheets were stored in phosphate buffered saline (PBS). For the experiments, samples were rinsed thoroughly with deionised water, cut into $5 \times 5 \mathrm{~mm}$ sized samples and air dried on calcium fluoride $\left(\mathrm{CaF}_{2}\right)$ coverslips.

\subsection{Two-photon microscopy}

A home built inverted two-photon microscope was used for both laser ablation and subsequent imaging (SHG and FLIM). A 20× NA 0.75 air objective (Nikon, Tokyo, Japan) was used to focus a $\sim 140 \mathrm{fs}, 80 \mathrm{MHz}$ (Chameleon Vision II, Coherent, Santa Clara, USA) pulsed Ti:sapphire laser to a single point onto the sample plane to create the cavities. The dependence of the collagen photodegradation on irradiation wavelength was investigated by varying the laser wavelength from $760 \mathrm{~nm}$ to $920 \mathrm{~nm}$ at constant $\sim 198 \mathrm{~mW}$ average laser output power (an equivalent power density of $\sim 14 \times 10^{6} \mathrm{~W} \mathrm{~cm}^{-2}$ ) for $10 \mathrm{~s}$. Vice versa, the power dependent effects on collagen at constant irradiation wavelength of $800 \mathrm{~nm}$ were studied by varying the irradiation power between $\sim 5 \mathrm{~mW}$ to $\sim 350 \mathrm{~mW}$. To determine the impact of laser heating around the focal spot, the sample was examined and compared after continuous and alternating on-off irradiations. In the former case, the sample was continuously exposed to laser irradiation for several seconds (i.e. $5 \mathrm{~s}, 10 \mathrm{~s}$ and $15 \mathrm{~s}$ ), whereas the latter process involved turning on and off the laser at $1 \mathrm{~s}$ intervals allowing the sample to cool down between each cycle. For imaging purposes, the femtosecond pulsed laser was tuned to $854 \mathrm{~nm}$ and the average excitation power was kept constant at $\sim 5 \mathrm{~mW}$ to avoid photodamage. The same $20 \times$ objective was used for collecting the back-scattered SHG and autofluorescence (AF) signals which were recorded with a photomultiplier tube and a TCSPC counting card (HPM-100-40 and SPC-150, Becker \& Hickl GmbH, Germany). A bandpass filter $427 / 10 \mathrm{~nm}$ (Semrock, New York, USA) was used to collect the SHG signal and $510 / 42 \mathrm{~nm}$ for detecting the AF signal. For pSHG imaging, a $\lambda / 4$-plate was placed in the input laser beam path at 45 degrees to the laser polarisation to produce circularly polarised light. The excitation polarisation was then selected using a polariser mounted on a motorised rotating stage. No analyser was used in the detection path and images were recorded with 8 different excitation polarisations from 0 to 180 degrees. 


\subsection{Raman spectroscopy}

InVia Raman microscope (Renishaw, New Mills, Gloucestershire, UK) with Raman StreamLine ${ }^{\circledR}$ imaging mode was used to perform chemical characterisation. The microscope was controlled by Renishaw's Wire 3.4 software. A $785 \mathrm{~nm}$ diode laser focussed by a $50 \times$ NA 0.75 air objective (Leica) was used with a 600 lines per mm grating centred at $1320 \mathrm{~cm}^{-1}$. The sample was imaged with $100 \mathrm{~mW}$ laser power in the sample plane and $30 \mathrm{~s}$ exposure to obtain reasonable signal-to-noise ratio (SNR). Using the Pearson correlation coefficient as the similarity function, $k$-clustering was performed to retrieve the spectrally different areas on the sample. Distribution maps were created by fitting the centroid set to the original spectral image as shown in Fig. 1S, ESI. $\dagger$

\subsection{Scanning electron microscopy}

Following laser irradiation and subsequent optical imaging, all samples were imaged with a FEI XL30 FEG scanning electron microscope (FEI, Eindhoven, Netherlands) operated at $5 \mathrm{kV}$. The samples were mounted on aluminium SEM stubs (Agar Scientific, Essex, UK) and then coated for $1 \frac{1}{2}$ minutes in an $\mathrm{Au} /$ Pd sputter coating system (Polaron E5000 Sputter Coater (Quorum Technologies Ltd, East Sussex, UK)) at $1.25 \mathrm{kV}$ and maintaining a current of $20 \mathrm{~mA}$ by adjusting the argon leak valve.

\subsection{Image analysis}

The SHG, AF and SEM images of the cavities were analysed with Image ${ }^{29}$ to determine the diameter of the cavity, the size of the different zones and the mean intensity of each zone. All measurements were repeated for 6 samples to determine the mean and the standard deviation. The FLIM images were processed with TRI2 software $^{\mathbf{3 0}}$ where a tri-exponential decay model was fitted for each pixel and the average lifetime calculated. The p-SHG data was processed by taking a Fourier transform of the SHG intensity as a function of the excitation polarisation angle for each pixel. The tensor ratios $\left(\chi_{13} / \chi_{15}\right.$ and $\left.\chi_{33} / \chi_{15}\right)$ and the main molecular orientation angle were derived from the amplitudes and the phase of the Dirac delta peaks respectively. ${ }^{31}$ The AF and SHG radial profiles were used to determine different centrosymmetrical zones surrounding the irradiation spot. The first zone was defined as the central area before the AF rises, the second zone corresponds to the AF ring and the third zone is the altered SHG signal.

\section{Results and discussion}

\subsection{Laser ablation of collagen}

Below $6 \mathrm{~mW}$ average laser power, equating to power density of $\sim 0.4 \times 10^{6} \mathrm{~W} \mathrm{~cm}^{-2}$, there was no observable damage to the collagen matrix. Above this threshold, an almost circular cavity was created at the focal spot which was likely due to multiphoton ionization process from femtosecond pulsed irradiation that resulted in plasma mediated laser ablation. ${ }^{32,33}$ In a clinical setting, the power density of the laser used varies depending on the application. For example in balloon catheter ablation $\sim 7.6$
$\mathrm{W} \mathrm{cm}{ }^{-2}$ is used, ${ }^{34}$ in most gynaecologic procedures using the $\mathrm{CO}_{2}$ laser the power density ranges from 8 to $12 \times 10^{3} \mathrm{~W} \mathrm{~cm}^{-2},{ }^{35}$ whereas in brain tissue ablation the power density of 1 to $3 \times$ $10^{9} \mathrm{~W} \mathrm{~cm}^{-2}$ is used. ${ }^{36}$

Hompland $\mathrm{T}$ et al. have demonstrated the use of SHG imaging to reveal the collagen content in various tumourous tissues and the loss of SHG signal can be resulted from the alteration of the triple helix structure at the fibrillar level rather than molecular denaturation. ${ }^{37}$ Thus, in our study, the loss of SHG signal at the ablated area (Fig. 1(a)) indicates the structural changes within the collagen fibrils caused by laser irradiation. Interestingly, a fluorescence ring was seen along the inner boundary of the laser induced cavity (Fig. 1(b)). This fluorescence zone is likely to be due to enhanced crosslinking of collagen molecules during laser irradiation. ${ }^{38}$ SEM image of the cavity (Fig. 1(c)) shows topographical alterations throughout the irradiated site. The characteristic D-banding periodicity, however, can still be seen along the boundary and away from the cavity (Fig. 1(d)). It is also interesting to note that the orientation of the fibrils around the cavity follows a tangential ordering. Although any laser induced remodelling of the scaffold in the present non-physiological conditions is unlikely, mechanical stress generated during the ablation process may be responsible for this apparent ordering. This hypothesis would however need further exploration. The effects of the laser power, wavelength and irradiation time on the size of the damaged site were quantified by measuring the diameter of the cavities revealed in the SHG, AF and SEM images.

Collagen photodegradation was found to be most sensitive around $800 \mathrm{~nm}$ excitation wavelength, signified by a larger cavity diameter compared to that induced by other wavelengths as seen in Fig. 1(e) and thus, inferring that the degree of collagen degradation is irradiation wavelength dependent. This is likely to be attributed to the direct photoabsorption of collagen. During this process, primary free radicals of water and organic molecules are targeted, inducing generation of secondary free radicals. These, in turn, interact with proline followed by glycine residues of the protein, causing dramatic alteration in the non-centrosymmetric structure of collagen. This results in the loss of hydrogen bonding network followed by the triple helix cleavage. Metreveli et al. proposed similar destabilization mechanism on studying laser irradiation effect on the collagen solution using calorimetric method. ${ }^{39}$

The diameter of the photodamaged region seems to be directly correlated to average laser output power. With the increase in the laser power, an exponential rise in the cavity diameter was observed through SHG and AF imaging (Fig. 2). At $134 \mathrm{~mW}$ average laser power, the diameter of the degraded SHG and $\mathrm{AF}$ regions were $1.74 \pm 0.25 \mu \mathrm{m}$ and $0.86 \pm 0.16 \mu \mathrm{m}$ respectively, whereas at $343 \mathrm{~mW}$ average laser power, the diameters were significantly increased to $39.8 \pm 1.86 \mu \mathrm{m}$ and $31.2 \pm 1.75 \mu \mathrm{m}$ respectively, elucidating the thermal effect of multiphoton absorption process.

An increase in the size of the degraded region was observed after $10 \mathrm{~s}$ and $15 \mathrm{~s}$ of continuous irradiation compared to that of equivalent total short irradiation intervals with interjecting cooling down periods. However, there was no significant 

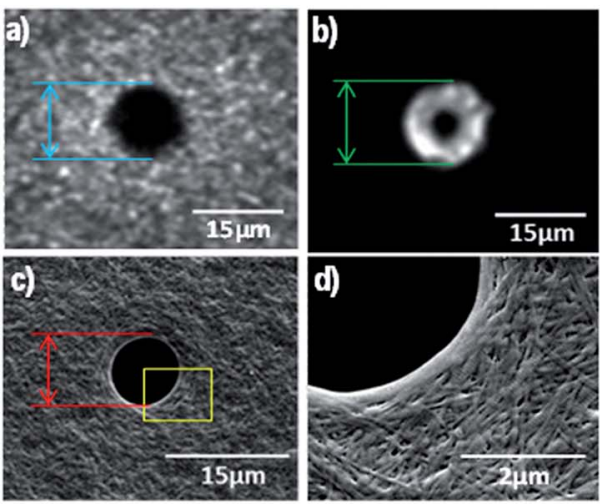

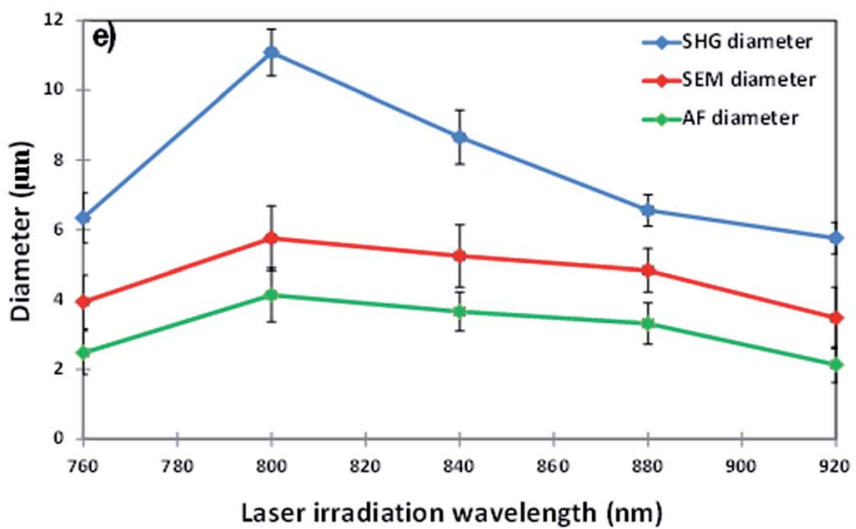

Fig. 1 SHG image (a), AF image (b), SEM image of the laser induced cavity (c) obtained at the resonance wavelength (800 $\mathrm{nm}$ ), and magnified SEM image ( $d$, highlighted area in $c$ ) showing the banding periodicity along the border of the cavity and surrounding areas of the laser irradiated site. SHG, SEM, AF cavity sizes for different irradiation wavelengths, $198 \mathrm{~mW}$ average laser output power and $10 \mathrm{~s}$ irradiation time (e).

difference observed with $5 \mathrm{~s}$ irradiation time (Fig. 3(a) and (b)). This result highlights the involvement of thermal effects where heat was generated during the interaction between highly intense femtosecond laser and the collagen matrix, disrupting surrounding tissues through conduction. Alternating on-off laser irradiation resulted in lesser structural damage compared to equivalent continuous irradiation as the off periods allowed for some heat dissipation and the stabilisation of the collagen matrix.

\subsection{Fluorescence lifetime}

To visualize the formation of the laser induced cavity, time lapse imaging was performed by incrementing the irradiation time in steps of $0.05 \mathrm{~s}$ (ESI $\dagger$ ) where thermal degradation of collagen followed by stabilisation can be observed. The cavity can be seen forming at the irradiation site from the exposure time above $\sim 0.7 \mathrm{~s}$. Due to photo-modification and thermal denaturation of the collagen structure, the cavity size increased rapidly and became stable at the exposure time exceeding $\sim 33 \mathrm{~s}$. A similar time dependent experiment performed by Hovhannisyan et al. showed that the fluorescence signal vanished due to photobleaching of fluorophores with increased illumination time. ${ }^{40}$ In our study, a fluorescence ring was always observed, with the ring thickness increasing with irradiation time, indicating increased crosslinking around the cavity.



Fig. 2 SHG diameter and AF diameter with varying laser output power on logarithmic scale showing exponential growth in cavity size observed at $800 \mathrm{~nm}$ irradiation (resonance) wavelength and $10 \mathrm{~s}$ irradiation time. 

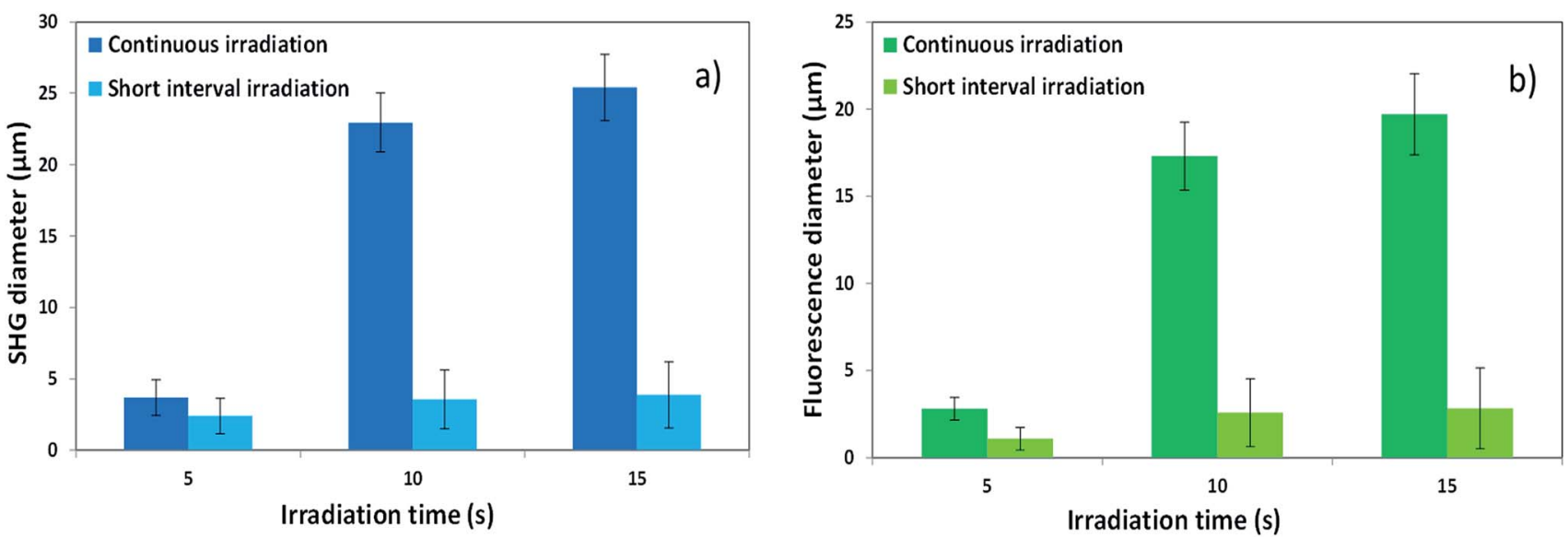

Fig. 3 SHG diameter (a) and fluorescence diameter (b) as a function of laser irradiation time showing the effects of continuous and short interval irradiation with $\sim 198 \mathrm{~mW}$ laser power and $800 \mathrm{~nm}$ irradiation (resonance) wavelength.

The fluorescence lifetime imaging (Fig. 4(a)) shows that the native collagen region has the longest lifetime of $1.63 \pm 0.07 \mathrm{~ns}$, whereas the average lifetime surrounding the laser irradiated region appears to have gradually reduced towards the centre of the cavity. The fluorescence lifetime of native collagen lattice measured by Lutz et al. was $2.8 \mathrm{~ns}^{41}$ However, fluorescence lifetime is generally unique for each type of fluorophore and it is highly dependent on the direct microenvironment. Fluorescent amino acids, tyrosine, advanced glycation end products, phenylalanine, and pyridinoline crosslinks are likely to be the fluorophores present at the crosslink sites and the change in their fluorescence lifetimes might correspond to their alterations. $^{\mathbf{4 1}}$

\subsection{Quantitative approach to collagen scaffold behaviour}

The p-SHG results (Fig. 4(b)) show some changes in the tensor ratios within the area where reduced SHG signal was detected. However, this effect was not observed further away from the

\section{a)}


Fluorescence b)
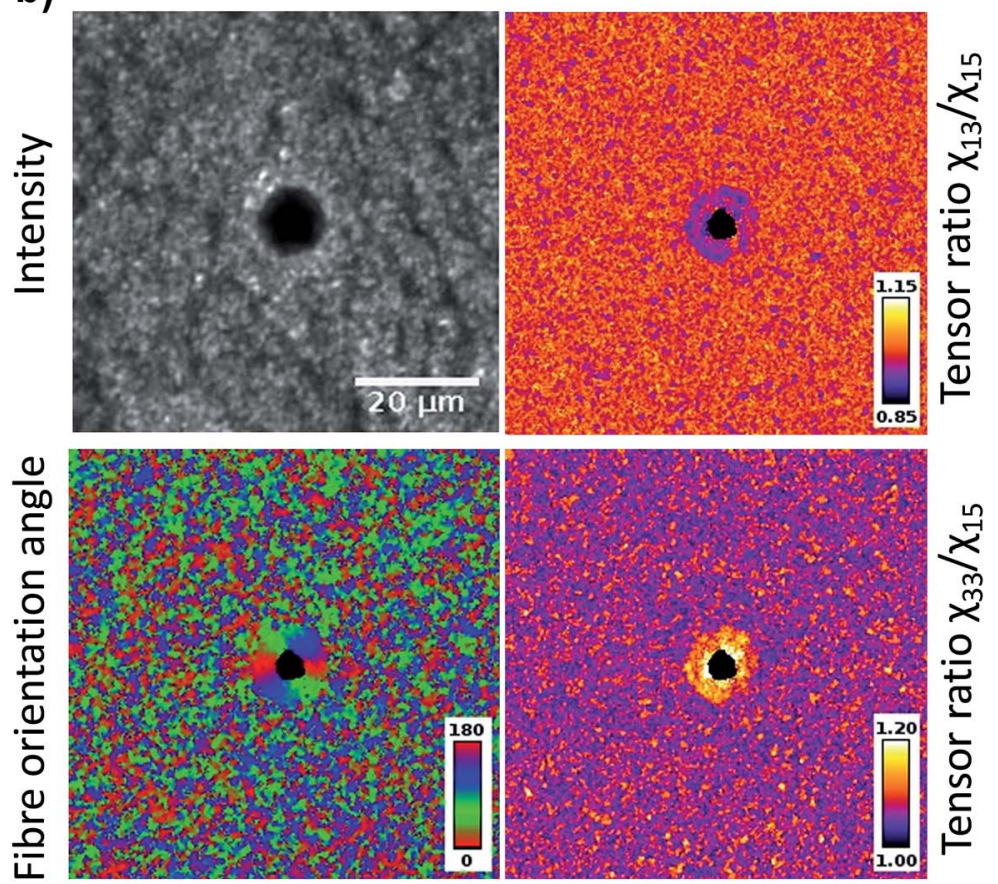

\section{Second Harmonic Generation}

Fig. 4 Autofluorescence intensity and average fluorescence lifetime (ns) (a). SHG intensity, main molecular orientation angle (degrees), tensor ratios $\chi_{13} / \chi_{15}$ and $\chi_{33} / \chi_{15}(b)$. 
a)
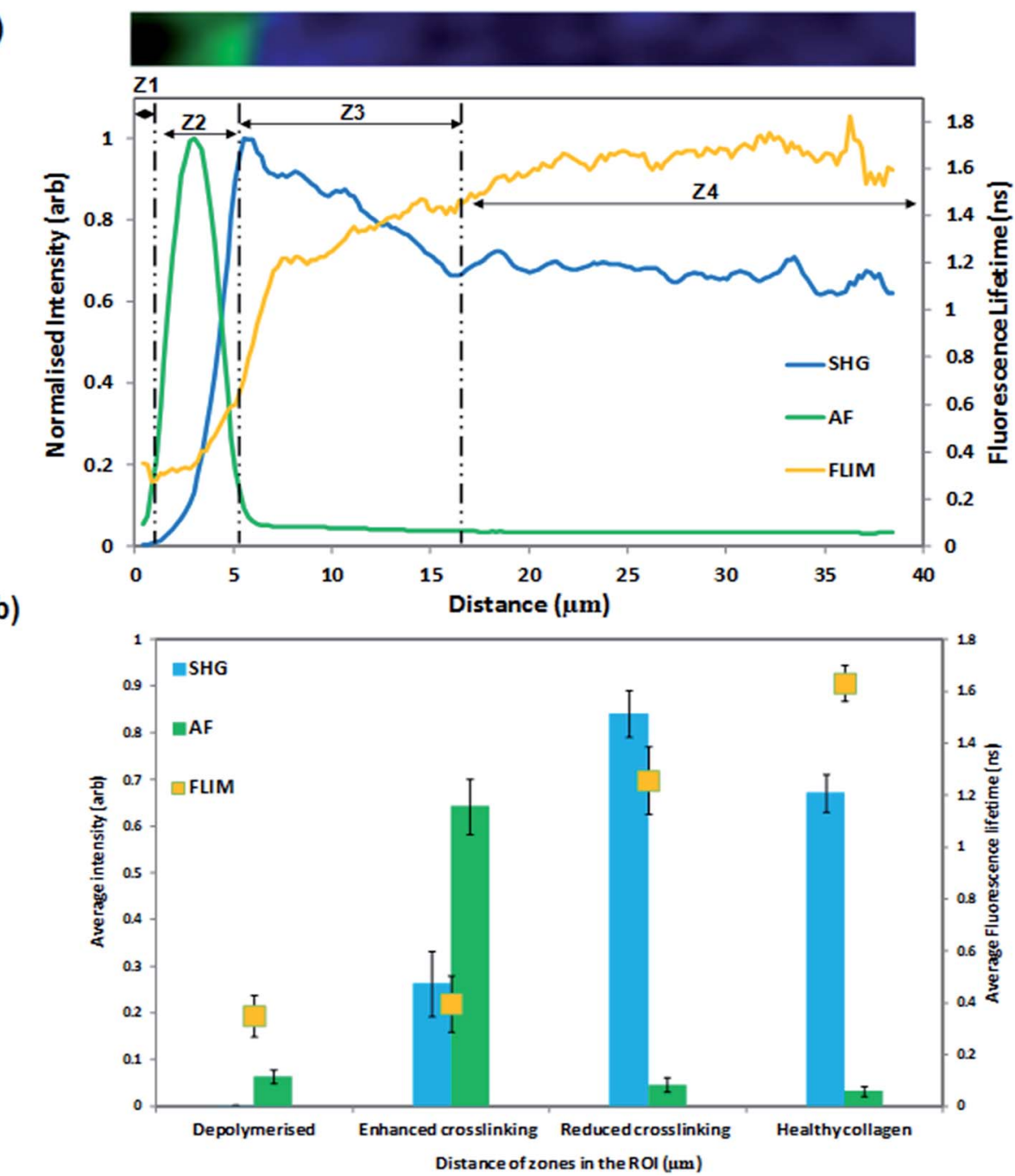

Fig. 5 Horizontal section of the combined SHG and AF image (top), and radial mean profiles showing the SHG, AF intensity variations and the average fluorescence lifetime (ns) in zone 1 (Z1), zone 2 (Z2), zone 3 (Z3) and zone 4 (Z4) over the region of interest (a). Average SHG and AF intensities and the average fluorescence lifetime in the four distinct zones (b).

cavity, indicating that the heat-induced degradation process did not alter the interatomic bonds in the collagen molecules. The p-SHG imaging shows that while the collagen molecules were randomly oriented in the gel, there was a high degree of molecular organisation around the border of the cavity. The collagen fibrils were precisely oriented in perpendicular to the radial direction from the cavity. Furthermore, the area where this organisation can be seen matches the area of increased AF, supporting the idea of ordered crosslinking of the collagen molecules around the irradiated zone.
Four distinct zones were identified from the SHG and AF images obtained at the resonance irradiation wavelength of 800 $\mathrm{nm}$ with an average laser power of $\sim 198 \mathrm{~mW}$ (Fig. 5(a)). Zone 1 represents the ablated area where collagen has lost its noncentrosymmetric structure by multiphoton absorption from water molecules. ${ }^{40}$ Zone 2 exhibits a thick AF ring extending outward from zone 1 and represents the zone of increased crosslinking associated with formation of new photoproducts, most probably involving tyrosine dimerization. ${ }^{40}$ Zone 3 shows an increased SHG signal compared to the intact gel and this

Table 1 Structural significance of the zones identified in the laser irradiated collagen matrix

\begin{tabular}{lll}
\hline Zones & Attribution & Structural significance \\
\hline Zone 1 & Depolymerised zone & Diffused featureless molecules with sparse collagen fibers \\
Zone 2 & Enhanced crosslinking zone & Intact covalent bonding with altered collagen triple helix \\
Zone 3 & Reduced crosslinking zone & Increased molecular interspaces with the triple helix structure maintained \\
Zone 4 & Healthy collagen & Unchanged non-centrosymmetric structure
\end{tabular}






Fig. 6 Raman spectra showing comparison between cluster 1 (depolymerised collagen), cluster 2 attributing to enhanced crosslinking zone, cluster 3 representing the reduced crosslinking zone and cluster 4 classified as healthy collagen.

Table 2 Characteristic Raman peaks of collagen $25,44,45$

\begin{tabular}{ll}
\hline Raman peaks $\left(\mathrm{cm}^{-1}\right)$ & Assignments \\
\hline 1668 & Amide I band \\
1454 & $\begin{array}{l}\text { Deformation vibrations in }-\mathrm{CH}_{3},-\mathrm{CH}_{2} \text { groups } \\
1310\end{array}$ \\
$1265-1244$ & Lipid molecules \\
1204 & Amide III band \\
1164 & Hydroxyproline in the triple helix structure \\
1082 & Pyranose ring (carbohydrate molecules) \\
1004 & Lipid molecules \\
& Phenylalanine
\end{tabular}

might be due to reduced collagen crosslinking which resulted in increased molecular interspaces. ${ }^{41}$ Heat conduction from the laser focal spot has resulted in the cleavage of covalent bonds in surrounding sites of laser ablated region, maintaining the triple helix structure.$^{\mathbf{4 0}}$ Finally, zone 4 represents randomly orientated non-irradiated collagen matrix exhibiting the longest fluorescence lifetime, as the control in this study.

The average SHG, AF intensities and the average fluorescence lifetime of each zone were quantified and compared as depicted in Fig. 5(b). In comparison to the native collagen gel, the $\mathrm{AF}$ intensity in zone 2 significantly increased by $\sim 78 \%$ and the SHG signal in zone 3 is $\sim 20 \%$ higher. The average fluorescence lifetime of the healthy gel was the longest at $1.63 \pm 0.07$ $\mathrm{ns}$ and gradually reduced to $1.17 \pm 0.13 \mathrm{~ns}$ in zone $3,0.37 \pm 0.14$ ns in zone 2 with molecular and hydrogen bond alterations and reaching the shortest lifetime in zone $1(0.31 \pm 0.08 \mathrm{~ns})$ where the collagen structures were completely altered. Table 1 summarizes the four optically and morphologically distinct zones identified using nonlinear optical multimodal imaging techniques. Natalia et al. studied collagen denaturation spread over laser irradiated patellar ligament (PL) tissue area using SHG, OCT and DSC. ${ }^{8}$ They suggested that supramolecular collagen structure undergoes various degrees of degradation resulting in four morphologically distinct zones all elliptical in shape and perpendicular to the collagen fibre long axis. The zones were identified qualitatively based on specific tissue architecture, cellular population and elliptical geometry: they were classified as zone 1 , homogenous; zone 2 , heterogeneous; zone 3 , crimped and zone 4 , distended tissue. ${ }^{8}$ Previous studies on laser ablated tissue characterization ${ }^{\mathbf{8 , 4 2 , 4 3}}$ were more of a qualitative approach which can be complemented by our study through quantitative zonal characterization of the laser irradiated collagen area.

Raman spectroscopy and k-clustering was used to analyse the photochemical alterations of 4 laser irradiated collagen gels alongside 4 non-irradiated control gels. Fig. 6 shows the Raman spectra of 4 identified clusters which correlate to the results obtained from AF and SHG. The significant Raman bands of collagen and their assignments are summarised in Table 2. In Fig. 6, cluster 1 (green) relates to the depolymerised zone (zone 1 ); cluster 2 (purple) relates to the enhanced crosslinking zone (zone 2); cluster 3 (blue) relates to the reduced crosslinking zone (zone 3) where the triple helix structure is maintained with increased SHG signal and cluster 4 (red) represents the native collagen corresponding to zone 4 . A similar study ${ }^{25}$ has been reported with dissolved type I collagen where amide III and I bands were suggested to be sensitive to conformational changes. We also observed a broadening of the amide III band at $1265 \mathrm{~cm}^{-1}$ in denatured collagen spectra, indicating the loss of the ordered triple helix structure. ${ }^{25}$ Alteration in the 1204 
$\mathrm{cm}^{-1}$ band may suggest the change in the molecular composition of the triple helix as it represents the presence of hydroxyproline (amino acid) in the helix. ${ }^{44}$ The high fluorescence component with completely lost Raman bands shown in Fig. 6 (depolymerised collagen) corresponds to the region close to the laser focal spot where the triple helical collagen was completely depolymerised. ${ }^{45}$ The Raman spectrum in the enhanced crosslinking zone as depicted in Fig. 6 has relatively high intensity and broader amide III band when compared to the healthy collagen and could be associated with the loss of intermolecular hydrogen bonds affecting the non-centrosymmetric property of collagen. $^{25}$

\section{Conclusions}

In conclusion, a multimodal optical approach was developed to study laser irradiated collagen degradation mechanism. The laser ablated collagen matrix was quantitatively classified into four distinct zones, namely the depolymerised zone (zone 1), enhanced crosslinking zone (zone 2), reduced crosslinking zone (zone 3 ) and the native collagen zone (zone 4) showing that laser irradiation results in different levels of tissue degradation. Previous models were more qualitative and were unable to morphologically assess collagen tissue modification. The collagen tends to lose its non-centrosymmetric structure at the focal spot due to direct laser ablation, followed by crosslinking along the inner border of laser induced cavity due to heat generated by multiphoton absorption and energy transfer. As heat conducts, reduced crosslinking occurs at the outer boundary, increasing intra and inter molecular spaces within collagen fibrils maintaining the triple helix structure resulting in increased SHG signal. The model was developed in our study with estimation for laser power threshold, optimal wavelength for controlled ablation and quantitative zonal characterization of the laser induced photo-disruptive effects. This can be extended to create engineering scaffolds for cell proliferation, intraocular surgery, cartilage reshaping, and skin rejuvenation. ${ }^{46-49}$ This multimodal nonlinear optical imaging approach might also be used as a potential tool for collagen rich tissue characterization providing a quantitative diagnostic tool with high accuracy, sensitivity and specificity compared to current techniques. ${ }^{\mathbf{8 , 5 0 , 5 1}}$

\section{References}

1 A. Bonifacio and V. Sergo, Vib. Spectrosc., 2010, 53, 314-317. 2 B. Brodsky and J. A. M. Ramshaw, Matrix Biol., 1997, 15, 545554.

3 N. Ignatieva, O. Zakharkina, I. Andreeva, E. Sobol, V. Kamensky and V. Lunin, Lasers Surg. Med., 2008, 40, 422-432.

4 F. O. Schmitt, C. E. Hall and M. A. Jakus, J. Cell. Comp. Physiol., 1942, 20, 11-33.

5 E. A. Chernoff and D. A. Chernoff, J. Vac. Sci. Technol., A, 1992, 10, 596-599.
6 R. Puxkandl, I. Zizak, O. Paris, J. Keckes, W. Tesch, S. Bernstorff, P. Purslow and P. Fratzl, Philos. Trans. R. Soc. London, Ser. B, 2002, 357, 191-197.

7 B. M. Kim, J. Eichler, K. M. Reiser, A. M. Rubenchik and L. B. Da Silva, Lasers Surg. Med., 2000, 27, 329-335.

8 N. Y. Ignatieva, A. E. Guller, O. L. Zakharkina, B. Sandnes, A. B. Shekhter, V. A. Kamensky and A. V. Zvyagin, Laser Med. Sci., 2011, 26, 401-413.

9 P. Stoller, P. M. Celliers, K. M. Reiser and A. M. Rubenchik, Appl. Opt., 2003, 42, 5209-5219.

10 C. H. Lee, A. Singla and Y. Lee, Int. J. Pharm., 2001, 221, 1-22.

11 J. T. Hashmi, Y. Y. Huang, S. K. Sharma, D. B. Kurup, L. De Taboada, J. D. Carroll and M. R. Hamblin, Lasers Surg. Med., 2010, 42, 450-466.

12 F. Loesel, J. Fischer, M. Götz, C. Horvath, T. Juhasz, F. Noack, N. Suhm and J. Bille, Appl. Phys. B: Lasers Opt., 1998, 66, 121128.

13 M. H. Niemz, Laser-tissue interactions: fundamentals and applications, Springer, 2007.

14 Y. Sun, W. L. Chen, S. J. Lin, S. H. Jee, Y. F. Chen, L. C. Lin, P. T. So and C. Y. Dong, Biophys. J., 2006, 91, 2620-2625.

15 C. A. Miles, T. V. Burjanadze and A. J. Bailey, J. Mol. Biol., 1995, 245, 437-446.

16 E. Leikina, M. Mertts, N. Kuznetsova and S. Leikin, Proc. Natl. Acad. Sci. U. S. A., 2002, 99, 1314-1318.

17 B. Eyden and M. Tzaphlidou, Micron, 2001, 32, 287-300.

18 D. Huang, E. A. Swanson, C. P. Lin, J. S. Schuman, W. G. Stinson, W. Chang, M. R. Hee, T. Flotte, K. Gregory and C. A. Puliafito, Science, 1991, 254, 1178-1181.

19 J. Schmitt, M. Yadlowsky and R. Bonner, Dermatology, 1995, 191, 93-98.

20 M. C. Pierce, R. L. Sheridan, B. Hyle Park, B. Cense and J. F. de Boer, Burns, 2004, 30, 511-517.

21 V. Crosignani, A. Dvornikov, J. S. Aguilar, C. Stringari, R. Edwards, W. W. Mantulin and E. Gratton, J. Biomed. Opt., 2012, 17, 116023.

22 S. Roth and I. Freund, Biopolymers, 1981, 20, 1271-1290.

23 Y. Tanaka, E. Hase, S. Fukushima, T. Yasui and T. Araki, Proc. SPIE, 2013, 8588, 85882X.

24 N. T. Wright and J. D. Humphrey, Annu. Rev. Biomed. Eng., 2002, 4, 109-128.

25 M. Wisniewski, A. Sionkowska, H. Kaczmarek, S. Lazare, V. Tokarev and C. Belin, J. Photochem. Photobiol., A, 2007, 188, 192-199.

26 Y. Yang, L. Gao, Z. Wang, M. J. Thrall, P. Luo, K. K. Wong and S. T. Wong, Proc. SPIE, 2011, 7903, 79032G.

27 S. L. Wu, H. Li, X. M. Zhang, W. R. Chen and Y. X. Wang, Laser Med. Sci., 2014, 29, 141-146.

28 R. A. Brown, M. Wiseman, C. B. Chuo, U. Cheema and S. N. Nazhat, Adv. Funct. Mater., 2005, 15, 1762-1770.

29 C. A. R. Schneider, W. S. Eliceiri and W. Kevin, Nat. Methods, 2012, 9, 671-675.

30 P. R. Barber, S. M. Ameer-Beg, J. Gilbey, L. M. Carlin, M. Keppler, T. C. $\mathrm{Ng}$ and B. Vojnovic, J. R. Soc., Interface, 2009, 6, S93-S105.

31 I. Amat-Roldan, S. Psilodimitrakopoulos, P. Loza-Alvarez and D. Artigas, Opt. Express, 2010, 18, 17209-17219. 
32 A. A. Oraevsky, L. B. Da Silva, A. M. Rubenchik, M. D. Feit, M. Glinsky, M. D. Perry, B. M. Mammini, W. Small and B. C. Stuart, IEEE J. Sel. Top. Quantum Electron., 1996, 2, 801-809.

33 A. Vogel, J. Noack, G. Hüttman and G. Paltauf, Appl. Phys. B: Lasers Opt., 2005, 81, 1015-1047.

34 V. Y. Reddy, P. Neuzil, S. Themistoclakis, S. B. Danik, A. Bonso, A. Rossillo, A. Raviele, R. Schweikert, S. Ernst and K.-H. Kuck, Circulation, 2009, 120, 12-20.

35 P. Soergel, M. Loning, I. Staboulidou, C. Schippert and P. Hillemanns, J. Environ. Pathol., Toxicol. Oncol., 2008, 27, 307-320.

36 S. Caroli and G. Záray, Analytical Techniques for Clinical Chemistry: Methods and Applications, John Wiley \& Sons, 2012, p. 486.

37 T. Hompland, A. Erikson, M. Lindgren, T. Lindmo and C. de Lange Davies, J. Biomed. Opt., 2008, 13, 054050.

38 K. M. Bottos, J. L. Dreyfuss, C. V. Regatieri, A. A. Lima-Filho, P. Schor, H. B. Nader and W. Chamon, J. Refract. Surg., 2008, 24, S715-S719.

39 N. Metreveli, L. Namicheishvili, K. Jariashvili, G. Mrevlishvili and A. Sionkowska, Int. J. Photoenergy, 2006, 2006, 4.

40 V. Hovhannisyan, A. Ghazaryan, Y. F. Chen, S. J. Chen and C. Y. Dong, Opt. Express, 2010, 18, 24037-24047.
41 V. Lutz, M. Sattler, S. Gallinat, H. Wenck, R. Poertner and F. Fischer, Skin Res. Technol., 2012, 18, 168-179.

42 A. D. Zweig, B. Meierhofer, O. M. Müller, C. Mischler, V. Romano, M. Frenz and H. P. Weber, Lasers Surg. Med., 1990, 10, 262-274.

43 K. M. Kirsch, B. D. Zelickson, C. B. Zachary and W. D. Tope, Arch. Dermatol., 1998, 134, 1255.

44 M. Gasior-Glogowska, M. Komorowska, J. Hanuza, M. Ptak and M. Kobielarz, Strain, 2010, 19, 20.

45 T. Nguyen, C. Gobinet, J. Feru, S. B. Pasco, M. Manfait and O. Piot, J. Spectrosc., 2012, 27, 421-427.

46 M. Oujja, S. Pérez, E. Fadeeva, J. Koch, B. Chichkov and M. Castillejo, Appl. Phys. Lett., 2009, 95, 263703.

47 L. P. Cunningham, M. P. Veilleux and P. J. Campagnola, Opt. Express, 2006, 14, 8613-8621.

48 S. Maruo and J. T. Fourkas, Laser Photonics Rev., 2008, 2, 100111.

49 V. Venugopalan, A. Guerra III, K. Nahen and A. Vogel, Phys. Rev. Lett., 2002, 88, 078103.

50 C. C. Wang, F. C. Li, R. J. Wu, V. A. Hovhannisyan, W. C. Lin, S.-J. Lin and C. Y. Dong, J. Biomed. Opt., 2009, 14, 044034.

51 I. Pavlova, K. R. Hume, S. A. Yazinski, J. Flanders, T. L. Southard, R. S. Weiss and W. W. Webb, J. Biomed. opt., 2012, 17, 0360141-0360149. 